\title{
An Investigation into the Dissipation of Vibrations Using Electromyography towards the Development of Self-Adapting Robotic Prosthesis
}

\author{
Morenike Magbagbeola ${ }^{1}$, Tijana Jevtic Vojinovic ${ }^{1}$, Mark Miodownik ${ }^{2}$, Stephen Hailes ${ }^{3}$, Rui C. V. Loureiro
}

\begin{abstract}
Vibrations can be used to convey positional or sensory information to prosthetic users. However, for the feedback to convey information consistently, daily fine-grained adjustments are required. This paper investigates whether vibration dissipation through the muscle can be tracked using EMG with the aim of providing reliable long-term sensory feedback. The results of this study showed that the magnitude of vibration artifacts can be measured using EMG and used to create a dissipation trend. This trend varies between participants but shows consistency when measured across multiple days. This novel way of measuring vibration dissipation can potentially be used as a basis of adaptive sensory control in future prosthesis studies.
\end{abstract}

Keywords-Vibrations, Artifacts, Dissipation, Electromyography, Prosthetics, Adaptive feedback, Robotics, Sensory substitution.

\section{INTRODUCTION}

The combination of accurate sensory feedback and effective prosthetic control provides better embodiment and functionality for prosthetic users [1]. This allows for finer motor control for complex tasks and an overall reduction of rejection rates for prosthesis, potentially leading to long term prosthetic use and treatment of neuropathic pain [2, 3].

Pattern recognition algorithms from EMG signals have been successfully used to improve motor control for high degree of freedom prosthesis, and the addition of sensory feedback can provide the missing proprioceptive component that closes the loop on effective prosthesis control [4].

Vibration is one of the most commonly used tools for providing sensory feedback to prosthetic users. Typically, it is used to provide information about grasping force; however, research has been conducted into its application as a modality

\footnotetext{
${ }^{1}$ Morenike Reni Magbagbeola, Tijana Jevtic Vojinovic are with the Aspire Centre for Rehabilitation Engineering and Assistive Technology (CREATe), University College London, United Kingdom (email: morenike.magbagbeola.16@ucl.ac.uk)

${ }^{2}$ Mark Miodownik is with the department of Mechanical Engineering, University College London, United Kingdom

${ }^{3}$ Stephen Hailes is with the department of Computer Science, University College London, United Kingdom

${ }^{1}$ Rui Loureiro is with the Aspire Centre for Rehabilitation Engineering and Assistive Technology (CREATe), University College London, United Kingdom (email: r.loureiro@ucl.ac.uk)
}

that provides texture, shape and proprioceptive information [5]. Several studies have shown task improvement and reduced cognitive load when elements of vibrational feedback were applied to the user compared to using vision-based feedback alone [6]. Moreover, it has been shown that effective sensory feedback can provide an increase in a sense of embodiment between the user and the prosthesis, making it easier to control $[7,8]$. One long term study conducted by Page et al, found that there was a significant level of embodiment of a prosthetic limb after either effective motor control or sensory feedback [9].

While current prosthetics can provide the necessary sensory feedback for effective use via vibration (or other modalities such as electrotactile or mechanical feedback), it was found that only about $20 \%$ of patients utilize such technologically advanced prosthesis [10]. While some of this is attributable to cost, a large proportion of prosthetic rejections are due to difficulties related to control, lack of embodiment and the long training times needed to interpret non-intuitive sensory information, all resulting in high cognitive load.

It has become apparent in the literature that the effect of sensory modalities, especially vibrations, varies between individuals and on a day-to-day basis for individuals due to physiological changes. For example, Mahns et al found that the sensitivity for vibrotactile stimulation is highest with maximum stimulations at $250 \mathrm{~Hz}$ compared to a lower sensitivity on hairy skin of $200-220 \mathrm{~Hz}$ [11]. Similarly factors such as fat, tightness of arm band, location of applied contact and sweat, not only effects perception of vibrotactile stimulation creating a sensory feedback mismatch [12] but also results in inconsistency in the EMG signal used for the prosthetic control. This means that while a specific perception (such as rough texture) can be applied by varying vibrational frequencies, the settings might not work for the user on another day or may be completely different to another user. Additionally, since vibrations themselves often add to the already noisy EMG signal, a continuous recalibration and adjustment of electrodes, stimuli and sensor placements are needed to ensure consistent perception of the feedback and retention of myoelectric control. This requires daily adjustments, high training time and potentially increases cognitive load every time the user wears the prosthesis. Peerdeman et al developed a survey that explored feedback needs for non-invasive sensory methods within literature and 
found that easy, intuitive and adjustable feedback were amongst the priorities for individuals [12] [13].

Despite the benefits attributed to sensory feedback in prosthesis, in order to allow for long term use of the prosthesis for control and rehabilitation outside of a laboratory; accurate, adjustable feedback needs to be continuously delivered over time. Therefore, a feedback system is needed that can account for physiological changes and create a uniform and consistent perceptual experience for users.

Wave propagation, such as those induced by vibrations, changes depending on the medium through which they travel. This may explain why physiological characteristics of an individual affects the perception of vibration specificity. Investigating how vibrations (or other stimuli) interact with the user and their relationship to how they are perceived may allow for a feedback system that accounts for the physiological characteristics.

Some studies have explored how vibrations propagate and dissipate on the surface of the skin and have shown it to generally behave as a decaying exponential function of distance from the source. They also found that the physiological characteristics of the skin, e.g. hair, affects the motor input due to the additional buffer between the stimuli and the cells responsible for picking up vibrations on the skin surface. This results in inconsistent perception of vibration. Additionally, the vibration sensors placed on the skin surface were not accurate enough to be able to distinguish how these physiological differences would affect propagation. The information gathered was primarily used to identify the best placement for vibration sources that alleviates the misperceptions associated from simultaneously applied stimuli [14] [15].

Looking at the artifacts in the muscle activity from EMG sensors may mitigate the inconsistencies that arises from physiological changes in and between participants. It is hoped that by correlating the dissipation to subjective tactile experiences, the feedback can be controlled and adjusted to maintain a consistent perception of vibration irrespective of the physiological characteristics of the user.

This paper reports on an initial study investigating the feasibility of using EMG sensors to measure vibration dissipation by recording the artifacts in the signal at measured distances from the stimuli. This is with the aim of exploring how this technique could be used in an adaptive sensory feedback system for robotic prosthesis in the future.

\section{METHODS}

\section{A. Experimental Design and Procedure}

Part I Design: 18 participants above the age of 18 (female 11, male 7) were recruited from University College London (UCL), with approval from the UCL ethics committee (Project ID: 14679/001), to assess how vibrations dissipate in the tissues surrounding vibration stimulus. All participants gave informed consent to the experimental procedure and the data from 15 participants was analyzed.

The main objective of the study was to record and assess the dissipation patterns induced by vibration stimulation using an electromyography (EMG) matrix on the dominant upper forearm. Vibration stimulation was achieved using eccentric rotating mass (ERM) motors encased in a customized 3D printed case. The motor sources were placed on the biceps and triceps of the dominant arm while an EMG matrix was placed equally spaced below the vibration source. Muscle contraction of the dominant arm triceps and biceps was also required and repeated using a period of 20 seconds contraction followed by 20 seconds of relaxation. Vibration dissipation and muscle contraction were recorded using surface electromyography while vibration frequency and duration were controlled using an Arduino UNO and power supply with a button switch.

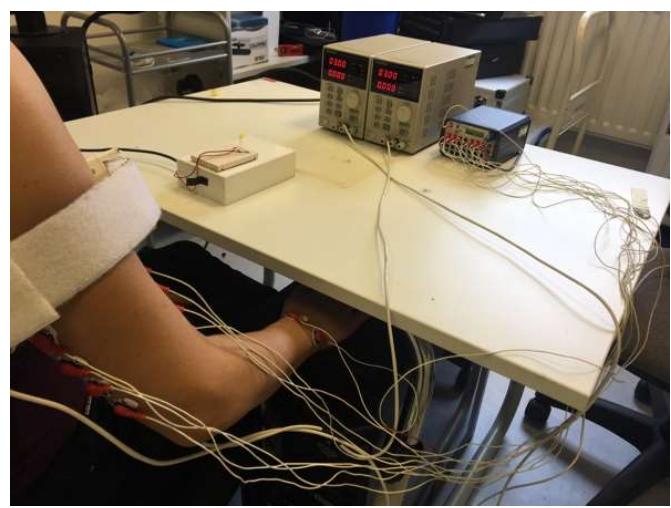

Figure I: Participant in Part I seated in resting position. Four unipolar electrodes are placed on both the biceps and triceps and connected to the TMSI Porti. Two vibration motors are placed on the top of the electrode array.

Part I Setup: The participants were seated comfortably at a table facing a computer screen and an LED interface. Their dominant upper limb remained in an "L-Shape" configuration whilst the non-dominant rested comfortably on their lap, as shown in figure I. The participants were instructed to place the hand of their dominant arm on a small cabinet located under the table so that when resting on the cabinet in the correct position, their lower arm was perpendicular to their upper arm. Participants were told at specific times to either push against the underside of the table to elicit triceps contraction or on top of the cabinet to elicit contraction of the biceps.

The computer screen displayed a simple graphical interface to warn of incoming vibrations and the LED display instructed participants to contract or relax the required muscle at the correct timing intervals. Two $34 \mathrm{~mm}$ Precision Microdrive vibration motors (model: 334-401) were placed on a velcro band and attached to the top of the biceps and triceps muscle belly of the upper dominant arm, approximately $15 \mathrm{~cm}$ from the elbow crease. Both motors were set to a frequency of approximately $25 \mathrm{~Hz}$. Four surface EMG electrodes were placed distally from each motor along the biceps and triceps approximately $2.5 \mathrm{~cm}$ apart from each other. This number of electrodes was to ensure enough data points were generated along the biceps to create a trendline. The ground and reference electrodes were placed on the wrist 
bone. EMG signals were acquired using the TMSi porti7 amplifier with a sampling frequency set to $512 \mathrm{~Hz}$ connected via Bluetooth to a laptop with open vibe software running for data collection.

Potential sources of variation were minimized by controlling the temperature of the room to approximately $20^{\circ} \mathrm{C}$, shielding the EMG electrode wires from electromagnetic noise and fixing them on a stable surface in a consistent way across subjects. Additionally, as previously described, electrode placement was consistent across participants.

Part I Procedure: Participants' body mass index (BMI) and upper arm circumference were recorded at the beginning of the session. Once a participant was seated comfortably in the correct position with the EMG sensors and vibration motors correctly placed, the experiment started. The experiment ran in four phases. Each phase required the participant to contract their muscle for 20 seconds and then to relax the muscle for an additional 20 seconds. This duration was to allow enough window length during post processing for analysis of a steady contracted or relaxed state [16]. This sequence was then repeated three times, beginning in the relaxed state. Each phase required a different muscle contraction sequence with either the motors above the biceps or triceps being continuously turned on for the duration of the phase. The four phases were: Biceps vibration with bicep contraction; biceps vibration with Biceps relaxation; Triceps vibration with Triceps contraction; and triceps vibration with triceps relaxation. Both biceps and triceps electrodes were observed and analyzed in each phase, resulting in eight conditions, as shown in table I.

TABLE I : Conditions table for Part I

\begin{tabular}{|c|c|c|c|c|}
\hline \multirow{2}{*}{$\begin{array}{c}\text { Vibration } \\
\text { Condition }\end{array}$} & \multicolumn{2}{|c|}{ Biceps Vibration } & \multicolumn{2}{c|}{ Triceps Vibration } \\
\cline { 2 - 5 } & Relax & $\begin{array}{c}\text { Biceps } \\
\text { Contract }\end{array}$ & Relax & $\begin{array}{c}\text { Triceps } \\
\text { Contract }\end{array}$ \\
\hline $\begin{array}{c}\text { Biceps } \\
\text { Electrodes }\end{array}$ & $\begin{array}{c}\text { Condition } \\
1\end{array}$ & Condition 2 & Condition 5 & Condition 6 \\
\hline $\begin{array}{c}\text { Triceps } \\
\text { Electrodes }\end{array}$ & $\begin{array}{c}\text { Condition } \\
3\end{array}$ & Condition 4 & Condition 7 & Condition 8 \\
\hline
\end{tabular}

Part II Design: Four participants above the age of 18 (females 3, male 1) were selected and recruited from University College London (UCL), with approval from the UCL ethics committee (Project ID: 14679/001), to assess changes in vibration dissipation over three days. All participants gave informed consent to the experimental procedure.

Differences in trend behavior were observed in part I of this study between participants. It is difficult to determine whether these differences are attributes of physiological differences between participants or an experimental flaw. Consistency of level of contraction, positioning of the participant in front of the table to ensure a 90-degree elbow flexion and interference of electrode wires were all identified to be potential sources of variability in experimental design.
Therefore, a second part to this study was devised addressing these issues and a small subset of the 18 participants were selected and dissipation trends analyzed over three days.

The vibrations were tested in a single frequency condition of approximately $25 \mathrm{~Hz}$ while a motor source was placed on the biceps of the dominant arm. An EMG array was placed equally spaced below the vibration source. Muscle contraction of the dominant arm was also required and repeated over a period of 20 seconds contraction and 20 seconds relaxation. Muscle contraction states were recorded using surface EMG and vibration dissipation was analyzed by assessing the magnitude of the vibration artifacts seen during data analysis. The vibration state was controlled using an Arduino UNO and a L298N motor driver.

Part II Setup: Participants stood facing an adjustable desk on which a computer screen and LED interface were placed. While standing straight, their dominant hand was resting parallel to the body by their side. The participants were asked to lift their arms so that the elbow joint was flexed at 90 degrees, measured using a goniometer, as shown in figure II. The adjustable desk was then raised until the participants' hand, in supination, was touching the underside of desk while the elbow remained at 90 degrees. This, in addition to lifting a known weight of $1023 \mathrm{~g}$, ensured that participants would maintain the same level of contraction throughout the experiment. The computer screen displayed a simple graphical user interface to instruct the participant to contract or relax their biceps at the correct timing intervals and to inform them of incoming vibrations. The LED display was an alternative method of instructing participants to contract or relax.
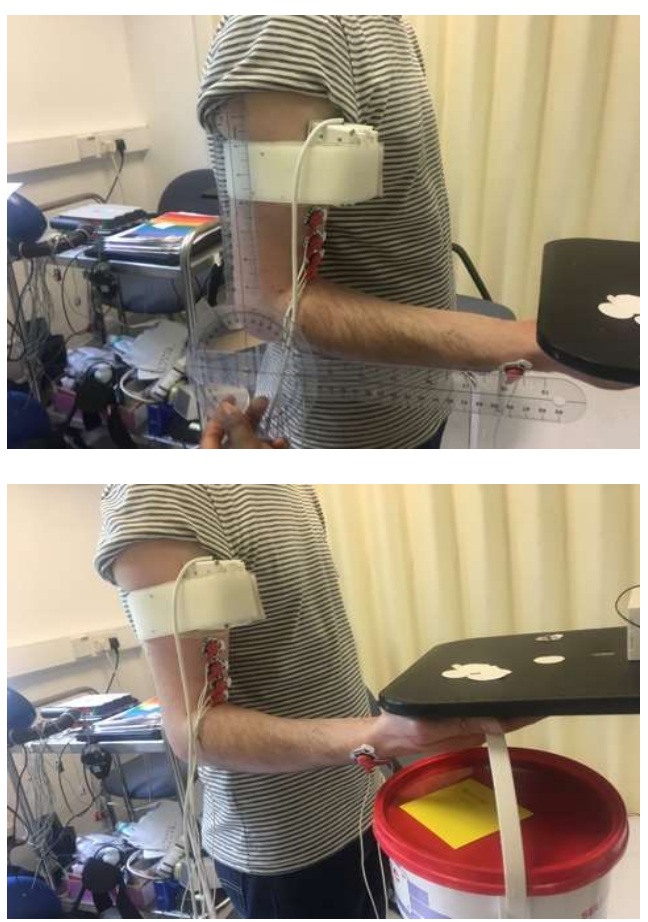

Figure II: Participant at 90 degree elbow flexion Part 2 in condition 3 and 4 as shown in Table TABLE II: Conditions for part II of the studyFour unipolar electrodes are placed under 

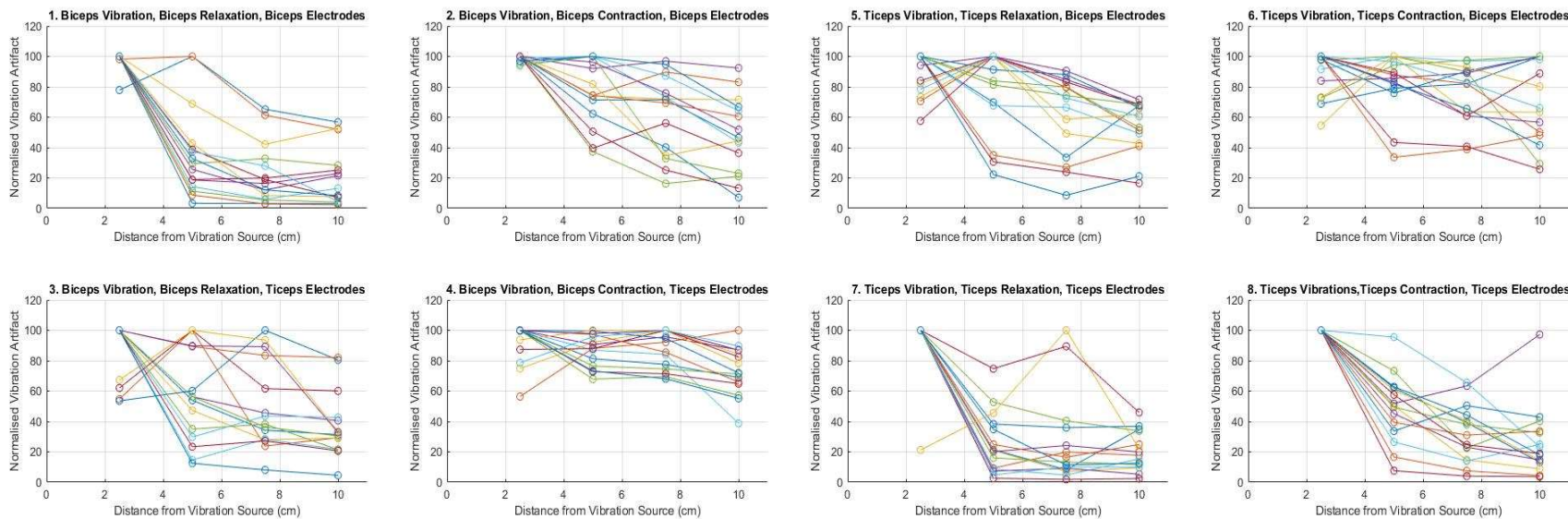

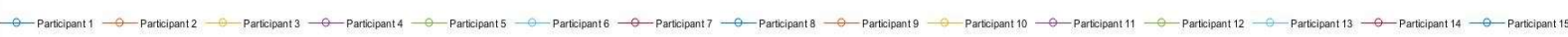

Figure III: Dissipation trend of each participant in the 8 conditions. Each coloured line represents a single participant. Results show some participants create outlier dissipation trends when compared to other participants in the same condition, e.g. participant 10 in condition 7. There is a general increase in variation of artifact magnitude the further from the vibration source. Conditions in with the vibration is ipsilateral to the electrodes $(1,2,7,8)$ the behavior of dissipation is similar. There is more variation across all electrodes when vibration is contralateral to the recording electrodes. The figure is in the same arrangement as the conditions in Table I.

As in Part I, a single Precision Mircodrive vibration motor (model: 334-401), was placed into a customized 3D printed case on a velcro band and attached to the top of the bicep muscle belly of the upper dominant arm, approximately $15 \mathrm{~cm}$ from the elbow crease. The vibration motor was set to a frequency of approximately $25 \mathrm{~Hz}$. Four surface EMG electrodes were placed distally from the motor along the biceps approximately $2.5 \mathrm{~cm}$ apart from each other. EMG signals were acquired using TMSi porti7 amplifier with a sampling frequency set to $512 \mathrm{~Hz}$ connected via Bluetooth to a laptop with open vibe software running for data collection.

Potential sources of variation were minimized by controlling the temperature of the room to approximately $20^{\circ}$, shielding the EMG electrode wires from electromagnetic noise and fixing them on a stable surface in a consistent way across subjects. Additionally, as previously described, electrode placement was consistent across participants and a known weight was lifted to normalized level of contraction across participants.

Part II Procedure: Participants' body mass index (BMI) was recorded at the beginning of the session. Once a participant was standing, holding the weight by their side with the EMG sensors and vibration motors correctly placed, the experiment started. The participant was required to contract their biceps by lifting the weight to the underside of the desk and holding it there for 20 seconds and then to relax the muscle for 20 seconds by holding the weight to their side. This process was then repeated three times, beginning in the relaxed state. The resulting four conditions, shown in table II, were: no weight/ no vibration; weight/no vibration; no weight/vibration; and weight/vibration. As with part I, arm circumference and room temperature were measured while electrode placement was measured and used to ensure consistent placement each day.
TABLE II: Conditions for part II of the study

\begin{tabular}{|c|c|c|}
\hline \multirow{2}{*}{$\begin{array}{l}\text { Vibration } \\
\text { Condition }\end{array}$} & \multicolumn{2}{|c|}{ Biceps Electrodes } \\
\cline { 2 - 3 } No Weight & Relaxation & Contraction \\
\hline Weight & Condition 1 & Condition 3 \\
\hline
\end{tabular}

\section{RESULTS}

\section{A. Data Analysis}

Data analysis was conducted in MATLAB using wellestablished functions and signal processing toolboxes. Statistical analysis was carried out using the statistical package IBM SPSS.
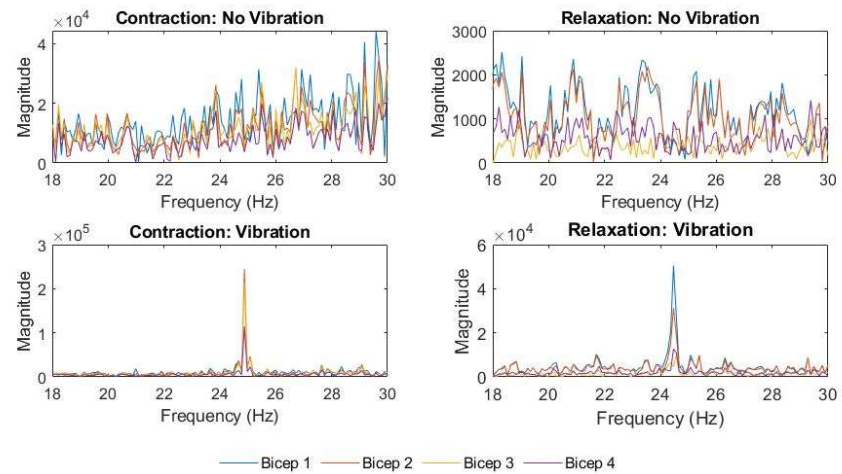

Figure IV: Fourier analysis of Relaxation and Contracted muscle states with and without vibration. The vibration artifact is orders of magnitude greater than the surrounding noise. 

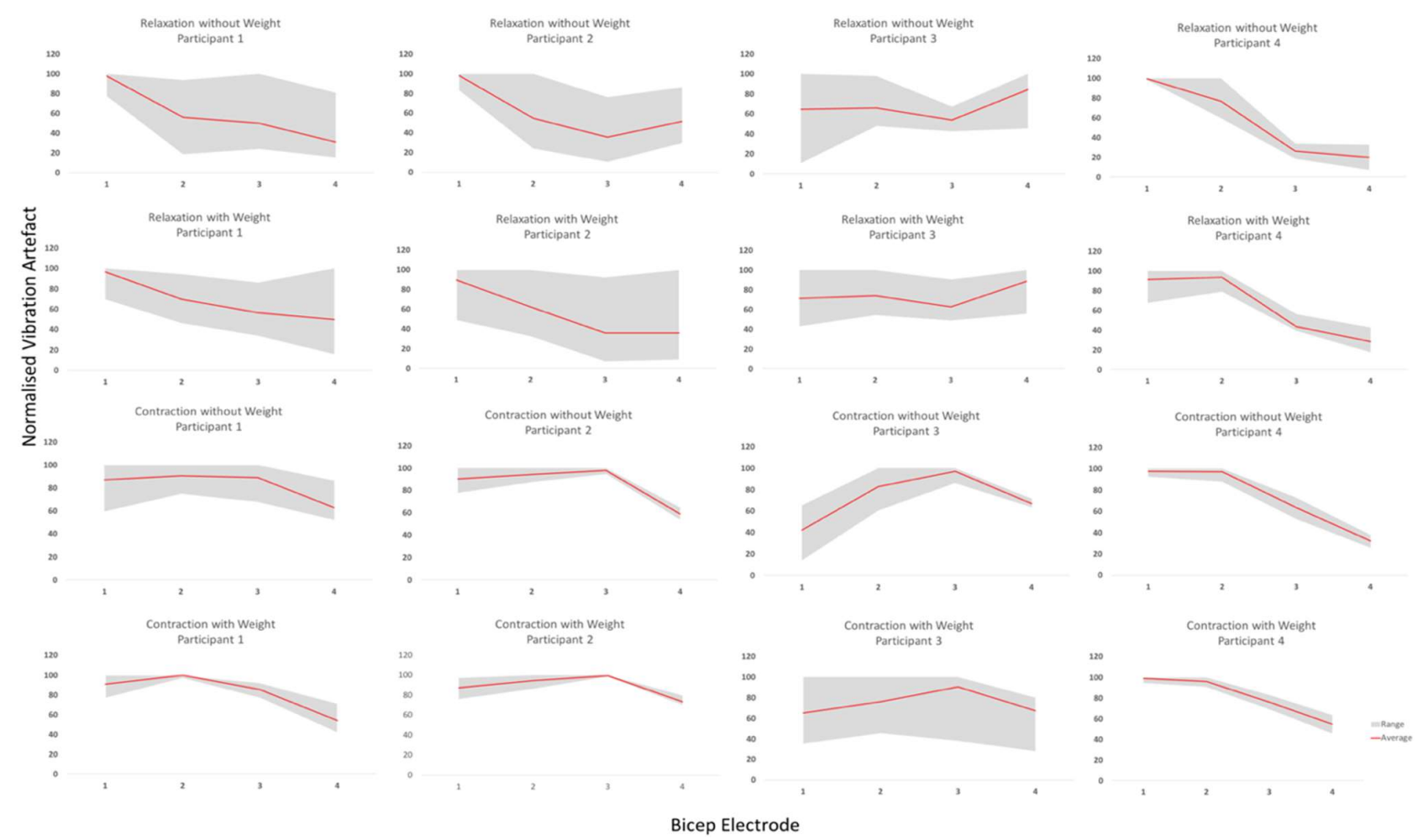

Figure V: Trend behaviour across multiple days for the four participants in every condition. The red line indicates the average value for that electrode while the grey indicates the range of values across the three days for that electrode. Each column corresponds to a participant.

Table III: Coefficient of Variation for each electrode across all participants in Part I

\begin{tabular}{lllll}
\hline Electrode & \multicolumn{1}{c}{1} & \multicolumn{1}{c}{2} & 3 & 4 \\
\hline Condition & & & & \\
$\mathbf{1}$ & 5.60 & 80.1 & 86.9 & 89.5 \\
$\mathbf{2}$ & 1.90 & 27.4 & 40.98 & 50.5 \\
$\mathbf{3}$ & 20.4 & 52.6 & 56.6 & 55.8 \\
$\mathbf{4}$ & 13.61 & 11.7 & 13.7 & 20.8 \\
$\mathbf{5}$ & 15.2 & 34.3 & 41.9 & 30.9 \\
$\mathbf{6}$ & 16.2 & 23.2 & 24.9 & 37.6 \\
$\mathbf{7}$ & 20.7 & 77.2 & 110.6 & 61.05 \\
$\mathbf{8}$ & 0 & 44.04 & 56.6 & 85.7 \\
\hline
\end{tabular}

\section{B. Time and Frequency Domain Analysis}

Both EMG time domain and joint time-frequency domain analyses were performed on the dataset. Peak analysis was conducted on the frequency spectrum to identify the magnitude of the vibration artifacts at specific frequencies across all electrodes.

The time domain analysis of vibration dissipation and muscle contraction or relaxation was measured using EMG electrodes during each phase. Different analysis techniques were used to compare magnitude of the vibration artifacts, including pwelch analysis, empirical mode decomposition and fast Fourier transforms. All confirmed the presence of the vibration artifacts at the correct frequencies and showed a decay in power at different electrode points. However, it was decided that Fast Fourier analysis was the most consistent analysis technique that could identify the vibration artifacts and produce dissipation trends based on them.

\section{Statistical Analysis}

To normalize the results across both parts of the experiment, the magnitude of the artifact for each electrode in one participant was normalised against the strongest magnitude for the same participant along all electrodes. This was done each time a trend was established.

The results of part I, displayed in figure III, show some inconsistency in overall trends across multiple participants for the averaged normalized magnitude. When analyzing visually, there is more consistent behaviour when the source of vibration is on the same muscle as the recording EMG electrode. For example, conditions 1, 2, 7 and 8 all have vibrations sources located on the same muscle as the recording EMG signals and all show relatively consistent behaviour across the 15 participants. In addition, the variation in magnitude increases as the distance to the vibration source increases, the degree of which varies between participants. Some participants exhibit completely different trend behaviour across all electrodes.

Visual inspection of the results from figure III seems to indicate consistency in trend behaviour with slightly less variability in the contracted condition. Conditions in which the electrodes are ipsilateral to the vibration source seem to produce a general logarithmic decay behaviour across most 
participants. To quantify this, the coefficient of variation (COV) was used, as defined in equation Eq.1, where $\mu$ is the mean and $\sigma$ the standard deviation of the averaged normalized artifacts for each participant. In part I, this produced a comparison across 15 distinct trends, the results of which are displayed in table III. The results show a general rise in variance the further away from the vibration source the electrode is. The smaller the individual number, the less variability there is across all participants for that electrode in the same condition.

Table IV : Intraclass correlation coefficient for each participant over the course of three days. The higher the value is to 1 the lower the variation across the three days and the more related the dissipation trends are.

\begin{tabular}{lcccc}
\hline Condition & 1 & 2 & 3 & 4 \\
\hline Participant & & & & \\
$\mathbf{1}$ & 0.7754 & 0.7261 & 0.7121 & 0.9498 \\
$\mathbf{2}$ & 0.7787 & 0.5422 & 0.9501 & 0.8983 \\
$\mathbf{3}$ & -0.115 & 0.2364 & 0.8488 & 0.0601 \\
$\mathbf{4}$ & 0.9619 & 0.9263 & 0.9899 & 0.9774 \\
\hline
\end{tabular}

TABLE V: Coefficient of Variance for each condition over multiple days in Part II. High COV values indicate large variation at the electrode across three days.

\begin{tabular}{lllll}
\hline Electrode & Bicep 1 & Bicep 2 & Bicep 3 & Bicep 4 \\
\hline Condition 1 & & & & \\
Participant & & & & \\
1 & 7.86 & 46.5 & 43.7 & 63.1 \\
2 & 5.74 & 43.4 & 72.8 & 38.4 \\
3 & 38.7 & 16.5 & 18.8 & 25.2 \\
4 & 0.831 & 17.5 & 20.4 & 52.8 \\
\hline Condition 2 & & & & \\
Participant & & & & \\
1 & 10.5 & 25.2 & 38.9 & 52.4 \\
2 & 22.6 & 46.9 & 76.7 & 81.1 \\
3 & 33.3 & 20.8 & 21.9 & 19.8 \\
4 & 14.1 & 10.6 & 15.1 & 41.1 \\
\hline Condition 3 & & & & \\
Participant & & & & \\
1 & 18.0 & 9.93 & 13.5 & 17.2 \\
2 & 7.69 & 6.10 & 2.56 & 7.21 \\
3 & 45.7 & 19.0 & 5.34 & 4.23 \\
4 & 3.26 & 4.13 & 9.81 & 12.4 \\
\hline Condition 4 & & & & \\
Participant & & & & \\
1 & 8.73 & 1.09 & 6.97 & 16.3 \\
2 & 9.47 & 5.45 & 0.63 & 4.24 \\
3 & 38.1 & 22.6 & 22.8 & 23.6 \\
4 & 2.04 & 4.46 & 7.37 & 10.08 \\
\hline
\end{tabular}

In part II, the COV was taken for each bicep across the three days for the averaged result of each participant each day. These results are displayed in table $\mathrm{V}$ and show that there is a generally smaller COV for both contracted conditions (conditions 3 and 4) across all biceps, consistent with visual inspection of figure IV. Of these two conditions it seems that contraction with weight produces a smaller variation of results over the course of three days for all participants.

In addition to the coefficient of variance, the Intraclass correlation coefficients (ICC) was taken for each participant in every condition for the three days in order to determine whether there was consistency in trend behaviour between participants or for each participant across multiple days. A two-way mixed effect, absolute agreement, multiple raters ICC was used by treating each day as a separate observer to an identical experiment. The results of this are displayed in table IV. These show significant intra-observer reliability for all conditions, similar to the coefficient of variance results. It is worth noting that, while the results for ICC show values of about 0.7 or above, which would typically indicate strong to excellent correlation [17], there is a general increase in the coefficient value for the different conditions. As with the $\mathrm{COV}$, the ICC shows much stronger correlation for the contracted condition ( 3 and 4 ), highlighted by the reduction in range in figure IV. This indicates that the trend in behaviour, though variable in some conditions, seems to remain the same for each participant over the course of several days.

\section{DISCUSSION}

In order to determine whether the proposed method of controlling sensorimotor feedback for prosthetic users is viable, this study investigated the feasibility of using EMG sensors to measure the dissipation of vibration.

We measured the magnitude of vibration artifacts observed after signal processing and developed a trend of behaviour based on the results. When measured across multiple participants, there was a large discrepancy in trend behaviour in all conditions. To determine whether these changes were due to individual differences or experimental inconsistencies, an additional experiment was conducted over a period of three days on a small subset of the previously recruited participants. This showed relative consistency in dissipation behaviour over the three days for each participant suggesting that the differences noted in the previous study were most likely due to individual differences in participant physiological characteristics.

The variability in relaxation condition is generally larger than in the contracted conditions. The conditions in which the electrodes are ipsilateral to the vibration source have lower variation between participants the closer to the source the electrode is placed. While the electrodes that are contralateral to the vibration source have slightly more consistent COV but vary significantly in trend behaviour. There is much larger variation overall for participant 3 in all conditions, this could possibly be due to the motor breaking and being replaced on day 3 of the experiment. 


\section{A. Implications for Design of an Adaptive sensory feedback system}

This method could potentially be useful in its ability to predict and define sensorimotor behaviour in myoelectric prosthetics. Given consistent electrode placement, the behaviour of vibration through participants' muscles is unlikely to change drastically on a day-to-day basis, especially in a contracted state. Though the physiological characteristics on the skin might affect the electrode contact, the uniformity of the differences across the electrodes will result in a consistent dissipation trend that can be calibrated against a previously established trend. This will allow factors that usually affect vibrotactile behaviour, such as sweat, hair, weight change and tightness of armband to be accounted for and adjusted based on the intensity and dissipation of the artifacts recorded and in a way that surface based vibration sensors are currently unable to do.

The biggest benefit to this method is that it exploits the EMG sensors used in prosthetic motion control. The technique to identify artifacts does not overlap with other control methods for myoelectric prosthetics and can be analysed through software only, adding little to no additional hardware for participants. The physiological characteristics would be consistent across all electrodes at the beginning of every recording cycle and the vibration artifacts are orders of magnitude greater than the usual noise attributed to EMG signals making them easily identifiable.

While the variation in trend is larger for the relaxed state, it is usually only during the contracted state that the myoelectric prosthesis is enabled. Subsequent experiments, conducted by our group, have shown consistent changes in dissipation trend behaviour when the frequency and intensity of the vibration stimuli is varied. Further work is currently being conducted that maps the unique subjective vibration experience to the corresponding dissipation trend for each participant. This will allow for a control system that calibrates the stimuli based upon the dissipation trend and not the subjective experience or previously identified parameters. Therefore, when perceiving different vibrations, from a system point of view, we are at an optimal level.

\section{B. Statistical analysis Discrepancies:}

This feasibility study does not allow us to determine an appropriate statistical analysis technique that would work for the entire data set and identify similarities between trends as opposed to differences. Initially a Kolmogorov-Smirnov normality test was conducted on the data set; this demonstrated that, when categorized by participant or condition, there was a mixture of normal and non-normal data sets for each participant. When categorized by day, all participants were analyzed as one group across all conditions and the data set produced a non-normal distribution. This is most likely due to the presence of non-normal data for some individual participants in some conditions. However, to determine trend behaviour, it is most appropriate to group the data by participant in different conditions. Statistically, to analyze data like this, either a one-way ANOVA or Kruskal Wallis test is used depending on the normality results. However, the data in each group does not always have the same distribution nor can we assume homoscedasticity which violates the assumptions of both tests.

As most non-parametric tests look for differences within different groups of data, it is difficult to determine statistically whether the distributions generated along each biceps electrode for each participant are similar to each other over the course of several days. Additionally, for part II, statistical tests to compare between participants would have been underpowered due to the low number of participants and high number of conditions. Furthermore, whilst most statistical tests are not appropriate for this study, the gold standard when comparing across multiple days is a minimum of three days. For these reasons, a correlation coefficient was considered to be the most appropriate means of assessing the data. The ICC test was chosen to look for similarities at each electrode across the three days as not only does it allow for multiple raters, in this case days, but also takes into account differences in the mean of variables [18].

\section{CONCLUSION}

A method to record and analyze vibration dissipation was presented. The method employs an EMG array of equidistantly placed sensors to identify signal artifacts produced by vibrations. Results show that there is consistency in trend behaviour across participants, with less variability in conditions in which the arm is contracted.

Based on these findings and the potential for this technique, we hypothesize that vibration dissipation through the muscles can be used to categorize and adjust sensory feedback for participants, creating a consistent feedback experience for long term prosthetic use. This will be the subject of future investigations.

\section{CONFLICTS OF INTEREST}

The authors declared no potential conflicts of interest with respect to the research, authorship, and/or publication of this article.

\section{ACKNOWLEDGMENTS}

The authors of this study would like to thank Dr. Henry Lancashire and Professor Norman Williams for their advice on statistical analysis. We would also like to thank participants who volunteered to take part in this study.

\section{REFERENCES}

[1] G. Dhillon and K. Horch, "Direct neural sensory feedback and control of a prosthetic arm," IEEE Transactions on Neural Systems and Rehabilitation Engineering, vol. 13, no. 4, pp. 468-472, 2005.

[2] P. W. Snow, R. C. Loureiro and R. Comley, "Design of a Robotic Sensorimotor System for Phantom Limb 
Pain Rehabilitation," in 5th IEEE RAS/EMBS International Conference on Biomedical Robotics and Biomechatronics, Sao Paulo, 2014.

[3] G. Rognini, F. M. Petrini, S. Raspopovic, G. Valle, G. Granata, I. Strauss, M. Solcà, J. Bello-Ruiz, B. Herbelin, R. Mange, E. D'Anna, R. Di Iorio, G. Di Pino, D. Andreu and Guirau, "Multisensory bionic limb to achieve prosthesis embodiment and reduce distorted phantom limb perceptions," Journal of Neurology, Neurosurgery \& Psychiatry, vol. 90, no. 7, pp. 833836, 2019.

[4] J. Liu, "Adaptive myoelectric pattern recognition toward improved multifunctional prosthesis control," Medical Engineering and Physics, vol. 37, no. 4, pp. 424-430, 2015.

[5] P. Svensson, U. Wijk, A. Björkman and C. Antfolk, "A review of invasive and non-invasive sensory feedback in upper limb prostheses," Expert Review of Medical Devices, 2017.

[6] H. Yamada, Y. Yamanoi, K. Wakita and R. Kato, "Investigation of a cognitive strain on hand grasping induced by sensory feedback for myoelectric hand," IEEE International Conference on Robotics and Automation (ICRA), pp. 3549-3554, 2016.

[7] R. B. Hellman, E. Chang, J. Tanner, H. Tillery and V. J. Santos, "A Robot Hand Testbed Designed for Enhancing Embodiment and Functional Neurorehabilitation of Body Schema in Subjects with Upper Limb Impairment or Loss," Frontiers in Human Neuroscience, vol. 9, no. February, pp. 1-10, 2015.

[8] M. D'Alonzo and C. Cipriani, "Vibrotactile Sensory Substitution Elicits Feeling of Ownership of an Alien Hand," PLoS ONE, vol. 7, no. 11, 2012.

[9] D. M. Page, J. A. George, D. T. Kluger, C. Duncan, S. Wendelken, T. Davis, D. T. Hutchinson and G. A. Clark, "Motor Control and Sensory Feedback Enhance Prosthesis Embodiment and Reduce Phantom Pain After Long-Term Hand Amputation," Frontiers in Human Neuroscience, vol. 12, no. September, pp. 1-16, 2018.

[10] T. R. Makin, F. De Vignemont and A. A. Faisal, "Neurocognitive barriers to the embodiment of technology," Nature Biomedical Engineering, vol. 1, pp. 1-3, 2017.
[11] D. A. Mahns, N. M. Perkins, V. Sahai, L. Robinson and M. J. Rowe, "Vibrotactile Frequency Discrimination in Human Hairy Skin," Journal of Neurophysiology, vol. 95, pp. 1442-1450, 2006.

[12] B. Stephens-Fripp, G. Alici and R. Mutlu, "A Review of Non-Invasive Sensory Feedback Methods for Transradial Prosthetic Hands," IEEE Access, vol. 6, pp. 6878 - 6899, 2018.

[13] B. Peerdeman, D. Boere, H. Witteveen, R. Huis in 'tVeld, H. Hermens, S. Stramigioli, H. Rietman, P. Veltink and S. Misra, "Myoelectric forearm prostheses: State of the art from a user-centered perspective," The Journal of Rehabilitation Research and Development, vol. 48, no. 6, p. 719, 2011.

[14] V. A. Shah, M. Casadio, R. A. Scheidt and L. A. Mrotek, "Vibration propagation on the skin of the Arm," Applied Sciences (Switzerland), pp. 1-12, 2019.

[15] K. O. Sofia and L. Jones, "Mechanical and psychophysical studies of surface wave propagation during vibrotactile stimulation," IEEE Transactions on Haptics, vol. 6, no. 3, pp. 320-329, 2013.

[16] T. Jevtic Vojinovic , T. Linley, E. A. Zivanovic and R. C. Loureiro, "Effects of Focal Vibration and Robotic Assistive Therapy on Upper Limb Spasticity in Incomplete Spinal Cord Injury," in IEEE 16th International Conference on Rehabilitation Robotics (ICORR), 2019.

[17] T. K. Koo and M. Y. Li, "A Guideline of Selecting and Reporting Intraclass Correlation Coefficients for Reliability Research," Journal of Chiropractic Medicine, vol. 15, pp. 155-163, 2016.

[18] J. Liu, W. Tang, C. Guanqin, L. Yin, F. Changyong and X. M. TU, "Correlation and agreement: overview and clarification of competing concepts and measures," Shanghai Archives of Psychiatry, vol. 28, no. 2, pp. 115-120, 2016. 